Archives

$8 \mid 1991$

Varia

\title{
Éléments d'histoire d'une grande entreprise textile de la Grèce contemporaine
}

\section{Christina Agriantoni}

\section{(2) OpenEdition}

\section{Journals}

Édition électronique

URL : http://journals.openedition.org/ccrh/2828

DOI : $10.4000 /$ ccrh.2828

ISSN : 1760-7906

Éditeur

Centre de recherches historiques - EHESS

Édition imprimée

Date de publication : 15 octobre 1991

ISSN : 0990-9141

Référence électronique

Christina Agriantoni, «Éléments d'histoire d'une grande entreprise textile de la Grèce contemporaine », Les Cahiers du Centre de Recherches Historiques [En ligne], 8| 1991, mis en ligne le 18 mars 2009, consulté le 22 avril 2019. URL : http://journals.openedition.org/ccrh/2828 ; DOI : 10.4000/ccrh.2828

Ce document a été généré automatiquement le 22 avril 2019

Article L.111-1 du Code de la propriété intellectuelle. 


\title{
Éléments d'histoire d'une grande entreprise textile de la Grèce contemporaine
}

\author{
Christina Agriantoni
}

1 L'historiographie grecque avait longtemps négligé le phénomène de l'industrialisation : la Grèce était «classée » comme un pays agricole, très en retard par rapport aux pays industrialisés, et cela au moins jusqu'à la Seconde Guerre mondiale. Cette schématisation ne rend compte de la réalité que partiellement. Car l'industrialisation s'est bien amorcée en Grèce, vers le dernier tiers du XIx ${ }^{e}$ siècle; pour l'étudier, l'échelle de l'économie nationale, pour autant que le mot ait un sens, est peut-être inappropriée. On devrait plutôt avoir recours à l'idée d'un type de renouvellement procédant par ruptures, par fragmentations et différenciations, géographiques et sectorielles. Cette réalité multiple reflète l'intégration des divers secteurs ou régions du pays dans les réseaux d'échanges, qui stimulent les économies locales à des niveaux et à des rythmes différents.

2 Premier «moteur » de cette intégration, le marché international des produits agricoles, auquel s'associent dès la fin $\mathrm{du} \mathrm{XVIII}^{\mathrm{e}}$ siècle les régions grecques dotées d'avantages comparatifs appréciables: il s'agit notamment de la frange côtière Nord-Ouest du Péloponnèse, où, par le relais des îles Ionniennes sous domination anglaise, se répand la culture du raisin sec, créant ainsi la première division de l'espace rural, entre régions de polyculture d'autoconsommation traditionnelle et régions de cultures marchandes d'exportation. Il y a ensuite les réseaux, aux frontières sans cesse repoussées plus loin, du commerce maritime, national et international, où les Grecs avaient conquis une bonne place, depuis le XviII ${ }^{\mathrm{e}}$ siècle également; ces réseaux, dont le trafic s'intensifie au XIX ${ }^{\mathrm{e}}$ siècle, animent certaines économies portuaires, notamment celle de l'île de Syros, qui monopolise tout au long de la première moitié du xix siècle le commerce de transit desservant la Méditerranée orientale. Un troisième réseau, dans un sens plus général, est représenté par les communautés grecques de l'extérieur, en territoire ottoman ou dans les villes européennes : il influe aussi bien sur le marché du travail que sur le marché de 
l'argent. Ces communautés florissantes offrent, d'une part, des débouchés aux hommes en surnombre (surtout ceux des régions montagneuses) en maintenant aussi vivace cette tendance à l'émigration, si typique des pays méditérranéens; et d'autre part, elles financent l'économie grecque, mais de manière également ponctuelle et inégale, soit par le moyen des dons des bienfaiteurs, soit, dans la seconde moitié du XIX ${ }^{e}$ siècle, par le moyen de l'extension de leurs activités bancaires dans le pays.

3 Ainsi, les changements de l'économie et de la société grecques au XIX ${ }^{\mathrm{e}}$ siècle dérivent de l'intégration, à des degrés divers, dans ces multiples circuits d'échanges, soit, en d'autres termes, du contact avec le monde extérieur. L'économie de marché qui se développe ainsi, longtemps qualifiée de "dépendante ", est en fait une économie résolument extravertie, qui se concentre de préférence sur le littoral et dans les grands ports du commerce extérieur. Et parce que les échanges externes sont l'élément le plus dynamique de ces changements, le secteur commercial de l'économie devient le lieu privilégié de l'accumulation des fortunes. Laissant loin derrière le monde de l'armement du $\mathrm{xvIII}^{\mathrm{e}}$ siècle, pour une bonne part détruit pendant la révolution nationale, ainsi que celui des anciens notables locaux, dépossédés de leur pouvoir local et investissant essentiellement l'appareil de l'État, laissant aussi le soin de la finance et de la banque aux mains des puissants Grecs de la diaspora, les nouveaux négociants "parvenus » de la génération d'après le renouveau formeront l'essentiel du monde des entrepreneurs de la Grèce moderne.

Ce sont eux qui vont se lancer dans l'aventure industrielle au cours du dernier tiers du XIX ${ }^{e}$ siècle. A cette époque en effet, certaines économies urbaines ont suffisamment mûri pour favoriser l'installation de petites industries de biens de consommation (alimentation et textile surtout). Evidemment, on ne peut pas parler de développement économique généralisé, encore moins de marché unifié à l'échelle nationale. Il s'agit plutôt d'une multitude de "poches", d'îlots d'économie d'échanges actifs, qui vont essayer par la suite, avec moins de succès que d'échecs par ailleurs, d'étendre leur rayon d'action. Dès à présent, en tout cas, une ville domine dans la hiérarchie urbaine: c'est Athènes, la capitale, lieu où s'entrecroisent tous les réseaux évoqués plus haut (ceux du commerce extérieur et de transit, par l'intermédiaire de son port, Le Pirée, ceux de la finance et de la banque), lieu de l'administration et du pouvoir politique, lieu, enfin, d'un marché de consommation en croissance continue (10.000 habitants au lendemain de l'Indépendance, 45.000 en $1870,107.000$ en 1889 et 167.000 en 1907).

5 Ces remarques introductives, très schématiques, ont simplement pour objet de situer le cadre des transformations que connaît l'économie grecque et qui déclenchent l'industrialisation, au cours des dernières décennies du XIX ${ }^{e}$ siècle. Ajoutons, sans pouvoir développer ce point ici, que les éléments évoqués, à savoir le fractionnement des secteurs et des régions, le primat commercial, le rôle capital de certains centres urbains et l'influence décisive des «aimants » externes, éléments qui façonnent la physionomie de la Grèce contemporaine, expliquent pour une large part le fait que cette industrialisation, au sens large, soit restée inachevée jusqu'à nos jours et qu'elle ait progressé en provoquant des distorsions extrêmes, dont le gigantisme athénien est l'exemple le plus frappant.

6 L'entreprise des Frères Retsinas dont nous allons esquisser à présent l'histoire dans ses grandes lignes, a été pendant de longues décennies le leader du textile qui fut à son tour la plus importante activité de l'industrie grecque. Cette entreprise, qui a fermé ses portes 
en 1979, a été la première à avoir mis à la disposition de la recherche historique son fonds d'archives, très prometteur pour les recherches à venir.

7 Pour les besoins de cette esquisse, on peut distinguer quatre étapes, en laissant de côté la période la plus récente, qui a abouti à la fermeture finale. La première, qui s'étend des origines (1872) jusqu'à la fin du siècle, est la période de l'ascension. Au cours de la seconde période qui couvre les trois premières décennies $d u x^{e}$ siècle, la firme ne connaît pas de changements majeurs, mais exploite à fond les acquis et certaines conditions favorables de l'époque. La troisième étape, les années 1930, est celle d'un élan nouveau brisé par la déclaration de guerre. La quatrième, enfin, la période 1945-1955, époque de la reconstruction, marquée par de grands bouleversements affectant l'économie et la société grecques et par de graves anomalies au niveau de l'entreprise, marque le début de son déclin.

8 Les origines de l'industrie cotonnière de la Grèce sont à situer dans la petite ville de Livadi, où se développe la culture du coton au temps de la crise américaine; mais son essor véritable, au cours des années 1870, est le fait de quelques centres urbains de première importance, tels que Le Pirée, Patras et Hermoupolis (île de Syros). L'entreprise des Frères Retsinas (Théodore, Alexandre et Athanase) est une des six filatures de coton fondées au Pirée à cette époque. L'origine de la famille est urbaine et probablement artisanale (le nom de la famille évoque l'exploitation de la résine des pins): Georges Retsinas, le père, venant d'Argos (ville du Péloponnèse-Est), était installé au Pirée depuis les années 1830 en tant que fabricant de boissons. Cette première installation, située au centre du Pirée, avantage considérable des premiers colons de cette ville nouvelle qui compte à peine 5.000 habitants au milieu du siècle, devait représenter par la suite une pièce essentielle de la fortune immobilière de la famille, servant surtout de gage pour les prêts hypothécaires des fils. Ainsi, par la valorisation des terrains, la croissance urbaine devait-elle financer, de manière indirecte, l'entreprise industrielle.

Les fils, c'est-à-dire pratiquement l'âné, Théodore, puisque c'est lui qui régnera sans partage sur les affaires de la famille jusqu'à sa mort en 1930, tentent leur chance dans le commerce piréote. Il n'est pas à exclure qu'au cours des années 1860, ils se soient occupés, entre autres, de l'exportation du coton (un bon tiers des exportations totales de cette matière se faisant par l'intermédiaire du Pirée), ce qui leur a permis de s'introduire dans le secteur cotonnier. Sans doute, les fonds investis dans l'industrie (achat du terrain du Pirée en 1871, construction de la fabrique et importation de l'équipement en 1872) proviennent-ils en grande partie de l'activité commerciale. Mais pas exclusivement : des fortunes hermoupolitaines y ont également contribué, puisque Théodore Retsinas se sert aussi de la dot qu'il a reçue de son mariage avec la fille d'un négociant de la ville insulaire, Aspasie Zografos. La Banque nationale, unique banque du pays à l'époque, a aussi joué un rôle certain dans l'affaire. Les Frères Retsinas ayant droit au crédit d'escompte ainsi qu'à un crédit compte courant gagé sur hypothèque, il est fort probable qu'à l'époque, ils s'en soient servis pour financer non seulement leurs fonds de roulement, mais aussi leurs premières immobilisations.

Il s'agit, au départ, d'une petite filature à vapeur de 5.000 broches, mais qui a coûté la somme de 600.000 drachmes environ, assez importante pour l'époque. La croissance fut rapide au cours des premières années, qui appartiennent à l'âge d'or pour l'ensemble du secteur : 7.000 broches en 1874, 10.000 en 1875. Les Frères Retsinas est déjà, à ce moment, la plus importante entreprise de la branche. Son expansion, par la suite, se fera largement aux dépends des autres, conformément à la loi du plus fort, dans un contexte de crises et 
de problèmes majeurs pour l'industrie naissante de la Grèce. Vers 1875 en effet, la dépression européenne touche les places grecques, et la grande crise orientale de 1875-1878, qui paralyse les échanges dans la région, met une fin prématurée à la perspective d'un débouché oriental pour la production industrielle du pays. Les filatures piréotes, encore pour quelque temps sur la lancée de leur dynamisme initial, réagissent en s'étendant vers le tissage. Retsinas participe à ce mouvement d'intégration verticale, d'abord prudemment, avec une cinquantaine de métiers mécaniques, puis plus résolument au cours de la brève reprise de 1879-1882. Mais le second coup, la crise de 1883-1885, première véritable crise industrielle de la Grèce, frappe durement les entreprises piréotes, dont près de la moitié ferment leurs portes. Retsinas lui, se trouve alors bien placé pour profiter de la situation. Il est vrai qu'il avait dès le départ fait preuve de mérites peu communs dans ce monde d'anciens marchands devenus accessoirement industriels. Il a le goût de la réclame, et il a su assurer une bonne direction technique à son affaire. Mais la raison essentielle de son succès, digne d'un véritable capitaine d'industrie style XIX ${ }^{e}$ siècle, et propre à confirmer les théories schumpétériennes sur le rôle de l'entrepreneur et de l'innovation, fut une percée rapide sur le marché local des tissus, avec un nouveau produit adapté aux habitudes locales: un tissu grossier et très solide, le drilli, massivement adopté par les couches populaires qui s'entassent à cette époque au Pirée et à Athènes. Cette cotonnade qui sera d'ailleurs bientôt appelée «le retsina ", du nom de l'entreprise, devait assurer à celle-ci une renommée solide et une place dominante sur le marché local, pour plusieurs décennies. Jusqu'à nos jours, les hommes de la maison conservent le souvenir de cette gloire ancienne, l'écho de ce «mythe fondateur » de la grande entreprise, légué d'une génération à l'autre.

11 En effet, fort de ce succès, Les Frères Retsinas devient alors une grande entreprise, la seule de ce rang dans un petit pays comme la Grèce. Entre 1887 et 1894, Retsinas rachète quatre autres entreprises textiles du Pirée qui avaient fait faillite, portant ainsi au nombre de cinq les usines composant son empire textile au Pirée, qui regroupe environ 25.000 broches, près de 500 métiers et plus de 2.000 ouvriers. Cette expansion est réalisée avec l'appui de la diaspora, dont le repli vers le centre national s'était amorcé à l'époque : depuis 1884, Retsinas s'était associé à Démosthène Hatzilazarou, homme d'affaires venant de Marseille et marié à sa soeur.

A partir de cette fin du XIX ${ }^{e}$ siècle, et jusqu'aux années 1920, Les Frères Retsinas va dominer l'industrie textile, de manière d'autant plus incontestable que celle-ci connaît, à la même époque, un développement extensif, avec la prolifération de petites firmes, le plus souvent spécialisées dans une fabrication bien précise (Hermoupolis compte par exemple 4 ou 5 fabriques de retordage de fils, destinés à un nombre analogue de fabriques de tricotage), tandis que les quelques firmes intégrées se concentrent au Pirée. Si donc, dans un premier temps, les pressions de la concurrence ont provoqué par réaction une tendance à la concentration, par la suite, et dans un contexte de protection assurée par la dévaluation de la drachme et par les tarifs douaniers, l'industrie grecque commence à épouser la physionomie qui sera la sienne pendant de longues décennies : une industrie de biens de consommation, faiblement mécanisée et préférant les techniques de main d'oeuvre et les structures familiales. La grande entreprise s'adapte à ce marché fragmentaire en faisant un peu de tout: la filature et le tissage bien sûr, mais aussi la couture des uniformes pour l'armée ou la teinture de fils et de tissus pour le compte d'autres producteurs. Elle coexiste ainsi harmonieusement avec cette nébuleuse de 
petites firmes, avec lesquelles elle noue parfois des liens qui sont en quelque sorte l'inverse de ce que l'on appelle aujourd'hui la sous-traitance.

13 A l'exception du renouvellement d'une partie au moins de l'équipement, qui dut avoir lieu entre 1905-1912, et du réaménagement des ateliers, réduits à trois, aucun changement notable n'intervient à cette époque. Au fil des ans, Les Frères Retsinas est devenu une véritable institution au Pirée, et son fonctionnement, une routine. En même temps, Théodore Retsinas, patriarche d'une grande famille dont les ramifications s'infiltrent dans le monde politique et bancaire, est devenu un grand notable du Pirée ; maire de la ville à deux reprises (1887 et 1891), il est un des rares représentants de l'industrie à entrer à la Chambre des députés en 1899-1902.

changements qu'apporte la longue période des guerres ne semblent pas bousculer immédiatement les habitudes. Ces changements sont d'abord l'extension des frontières (la Grèce annexe la Macédoine, l'Epire et la Thrace orientale à la fin des guerres balkaniques de 1912-13), et l'installation dans le pays de près d'1,2 millions de réfugiés de l'Asie Mineure (suite à la défaite de l'armée grecque en 1922 et à l'échange des populations). Les deux événements, entraînant une extension considérable du marché intérieur, ont singulièrement favorisé, au cours des années 1920, l'expansion de l'industrie, fortement soutenue en outre par une protection douanière renforcée: le déséquilibre financier que connaît la Grèce à cette époque, comme tous les pays débiteurs de l'entre-deux-guerres, oblige à la mise en place d'une véritable politique d'industrialisation par substitution aux importations. Ainsi la croissance de l'industrie se fait-elle de manière extensive, sur le modèle déjà établi, et reposant maintenant sur une main d'oeuvre abondante et bon marché.

Lorsque, en 1925, Les Frères Retsinas se transforme en société anonyme, avec un capital de 7 millions de drachmes partagé entre Théodore Retsinas et Démosthène Hatzilazarou, l'équipement des trois usines, dont l'une, la fabrique initiale, est une véritable citadelle occupant 4,7 hectares, semble plutôt vétuste, aucun investissement neuf n'ayant été effectué pendant près de quinze ans. Peut-être le vieillissement des fondateurs n'est-il pas indépendant de cette situation. A cette époque, Théodore Retsinas a presque cent ans, et pourtant il préside, jusqu'à sa mort en 1928, les réunions du Conseil d'Administration de l'entreprise, qui demeure une affaire strictement familiale (elle ne sera pas côtée en Bourse et, parmi les 38 actionnaires recensés en 1951, 22 sont des descendants directs de Théodore et de ses cinq frères et soeurs, les autres étant probablement des membres de la famille associés par mariage).

Avec la mort de D. Hatzilazarou en 1930 et l'avènement à la tête de l'entreprise de la génération des petits-fils de Retsinas, s'ouvre la troisième étape de son histoire, marquée par un effort de modernisation considérable. Celle-ci était en réalité impérative car cette même expansion des années 1920, butant sur la crise de 1929-32, avait préparé les conditions d'une concurrence menaçante. D'abord, avec l'annexion de la Macédoine, il faut désormais compter avec deux autres anciens foyers du textile, les villes de Salonique et de Naoussa. Ensuite, dans le climat favorable de la période, de nouveaux entrepreneurs, issus du monde des réfugiés ou de ceux qui avaient fait fortune par des spéculations en temps de guerre, s'introduisent dans le secteur cotonnier et fondent des entreprises promises à un bel avenir (comme Piraiki-Patraiki de Christos Katsambas). Des industriels d'Hermoupolis viennent également à cette époque s'installer sur le continent ( Aigaion et Velca sont fondées à cette époque). A la fin de la décennie glorieuse, le secteur 
est menacé par une crise de surproduction qui amène le gouvernement à imposer des restrictions, en 1932, à la création de nouvelles usines.

C'est donc dans ce contexte que Les Frères Retsinas. essaie de rattraper le temps perdu. Avec un total d'investissements neufs de 70 millions de drachmes, provenant presque en totalité des profits réalisés pendant les années 1927 à 1938 (dont 45,5 au cours des années 1931-35), la capacité des filatures est augmentée d'environ $40 \%$, une partie des métiers est remplacée et de nouveaux métiers automatiques sont installés, tandis que les vieilles machines à vapeur ou à pétrole sont remplacées par des moteurs électriques. Ainsi, à la veille de la Seconde Guerre mondiale, Les Frères Retsinas arrive à maintenir sa position en tête de l'industrie cotonnière, avec 38.000 broches et environ 1.000 métiers, chiffres qui représentent plus de $15 \%$ de la capacité totale de la branche, qui compte alors 89 entreprises. Mais on doit nuancer la portée de cette modernisation, car il semble bien qu'il y eut un héritage, sacralisé peut-être et en tout cas trop pesant, auquel on n'a pas voulu toucher, à savoir le type de production des usines: elles se limitent toujours à la fabrication des fils et tissus grossiers, comme c'est le cas pour l'ensemble de l'industrie cotonnière d'ailleurs, habituée à s'adresser à une clientèle de faibles revenus et laissant aux importations le marché des fils fins et des tissus doubles et de qualité supérieure.

Les documents de l'entre-deux-guerres permettent de se former une idée assez précise du monde des travailleurs de l'entreprise ; celle-ci, étant donné ses dimensions, est assez représentative de la classe ouvrière du Pirée. Comme il fallait s'y attendre, l'écrasante majorité de la main d'oeuvre se compose à cette époque de femmes, le plus souvent très jeunes : $90 \%$ en 1933. La présence massive des réfugiés ( $55 \%$ des ouvriers et ouvrières en 1933) confirme également que ce sont bien ces derniers qui ont soutenu l'industrialisation de l'entre-deux-guerres. L'effectif de cette population ouvrière, payée à la journée, n'est pas stable : il oscille en fonction du travail prévu pour la journée. D'autre part, tout au long de l'entre-deux-guerres et jusqu'à l'instauration du système de la Sécurité Sociale en 1937, une "Caisse de maladie-retraite" pour les travailleurs est financée par des sommes retenues régulièrement sur les salaires à la journée ainsi que par des «dons à la caisse » faits par l'entreprise, mais pour des montants variables. Un autre élément est enfin révélateur de la physionomie du monde ouvrier et indique sa grande mobilité : en 1940, 58 \% du personnel travaille à l'usine depuis moins de cinq ans.

19 Sans doute les difficultés des années de guerre ont-elles mis un terme à la dynamique ascensionnelle des années 1930. Il semble en effet que jusqu'en 1945, les usines aient travaillé au ralenti et qu'une partie de la production ait été confisquée par les forces d'occupation, tandis qu'en 1944 l'usine centrale fut gravement endommagée au cours des combats, à deux reprises, peu avant la Libération ainsi qu'au premier jour de la guerre civile. Pourtant la véritable césure, qui marque le début du long déclin de la grande firme, doit plutôt être située près d'une décennie plus tard, au milieu des années 1950.

20 C'est en effet au cours de la période critique de 1946-53 que le sort de la firme semble s'être joué. Au départ, et jusqu'en 1950, les bilans dégagent une tendance au redressement: si les créances passives sont en hausse (la firme s'endette, à court terme toujours), les valeurs d'exploitation et les profits bruts le sont aussi. Le renversement, entre 1950 et 53, est dramatique : le passif exigible est multiplié par trois, les profits bruts sont réduits de moitié et la firme accuse des pertes consécutives. En 1955, l'ensemble de créances passives dépasse l'actif réalisable et disponible, et les intérêts dus dépassent les profits bruts. On décide alors de procéder au démantèlement des deux usines, tandis que 
la société est mise sous contrôle judiciaire. En 1957, seule l'usine centrale travaille, avec 300 métiers et 6.200 broches.

21 Il est prématuré de proposer des explications valables de cette décadence. Les rares études qui existent sur cette période cruciale de l'histoire de la Grèce, et les bribes d'information qui sont pour le moment disponibles sur l'entreprise, nous permettent seulement d'avancer quelques hypothèses de travail. La première catégorie d'hypothèses est d'ordre économique. Les difficultés majeures en effet semblent venir du côté des ventes. Les années 1946-49 ont été en principe favorables aux industries de biens de consommation, appelées dans un premier temps à couvrir les énormes pénuries des années de guerre. A la même époque, ce sont précisément ces industries qui ont été de préférence soutenues par les crédits bancaires; l'ensemble du secteur achève donc sa reconstruction à la fin des années 1940 (en 1950, le volume de l'ensemble de la production industrielle avait retrouvé les niveaux de 1939). Or, au début de la décennie suivante, la demande ne suit plus les rythmes de l'offre. Les longues années d'inflation galopante (l'indice des prix à la consommation passant de 177 en 1946 à 418 en 1951, avec comme base $1939=1$ ) avaient épuisé le pouvoir d'achat, et surtout celui des couches populaires, auxquelles s'adresse la production de Retsinas (dans une proportion de $90 \%$, comme l'affirme un rapport de la firme). Qui plus est, une certaine facilité, voire l'euphorie de l'immédiat après-guerre, période de pénurie et de spéculations de toute sorte, avait détourné de tout effort de modernisation : l'essentiel des profits de la période 1946-50 (qui ont été jugés « exceptionnellement élevés " plus tard, la firme étant pour cela taxée $a$ posteriori) a été consacré aux réparations, et non pas à des investissements neufs. Ce n'est qu'en 1953, trop tard peut-être, qu'il fut question de remplacer quelques métiers par ceux de la nouvelle génération de l'après-guerre.

La seconde catégorie d'hypothèses serait plutôt d'ordre social. La guerre, et surtout la guerre civile qui a suivi (1946-1949), représente une énorme cassure, qui a définitivement brisé les rapports de type paternaliste style XIX ${ }^{e}$ siècle qui caractérisaient jusqu'à cette période la vie intérieure de l'entreprise. Nous ne pouvons pas avancer plus loin pour le moment. Nous pouvons seulement constater que divers documents de cette période dégagent un climat de méfiance, de malaise et de contestation sociale, latente sinon ouvertement exprimée, qui a peut-être pesé sur les chances d'une reprise durable.

23 Enfin, la dernière catégorie d'hypothèses, d'ordre social et politique, concerne les changements qui interviennent au niveau des couches dirigeantes après la guerre, et qui représentent aussi une rupture. Le renouvellement du personnel politique et le changement des puissants " protecteurs » externes (les Américains prenant le relais des Anglais à partir de 1947 et intervenant de manière directe dans la gestion économique), correspondent à des bouleversements notables au niveau des élites sociales et économiques. La bourgeoisie d'affaires de l'entre-deux-guerres a été, pour une bonne part, mise à l'écart par des entrepreneurs d'un type nouveau, souvent d'origine douteuse, qui ont su exploiter les anomalies de la guerre ou les secteurs à gros profit de l'après guerre, comme les travaux publics ou le bâtiment. Chez Retsinas, l'homme fort des années 1930 ayant disparu pendant la guerre, les nouveaux dirigeants, toujours des membres de la famille et probablement moins rompus aux affaires (est-ce la règle de la troisième génération qui se confirme ?), ne semblent en tout cas avoir ni le même accès facile aux couloirs du pouvoir ni la même puissance sociale que leurs prédécesseurs.

24 Les Frères Retsinas n'a jamais retrouvé la grandeur du passé après cet echec de la période 1945-55. L'entreprise a vivoté au cours des années 1960, tandis qu'un effort de 
redéploiement en province, entrepris en 1975 (création d'une filiale à Aigion, dans le Péloponnèse), ne l'a pas sauvée de la fermeture finale. Aujourd'hui l'usine piréote, le dernier vestige d'une longue histoire, s'apprête à disparaitre sous les bulldozers. D'une certaine façon, avec elle disparaît tout un monde : un certain capitalisme industriel né à la fin du XIXe siècle, qui s'est épanoui dans l'entre-deux-guerres, mais qui n'a pas vraiment pris racine en Grèce.

\section{AUTEUR}

\section{CHRISTINA AGRIANTONI}

Christina AGRIANTONI est chercheur au Centre de Recherches néohelléniques d'Athènes ; elle a été directeur d'études associé à l'EHESS en 1991. 